\title{
Les territoires autochtones face aux industries extractives au Chili : Enjeux environnementaux et modes de résistance communautaire
}

Blaise Pantel et Ximena Cuadra Montoya

\section{(2) OpenEdition}

\section{Journals}

Édition électronique

URL : http://journals.openedition.org/ere/511

DOI : 10.4000/ere.511

ISSN : 2561-2271

Éditeur

Centr'ERE

Référence électronique

Blaise Pantel et Ximena Cuadra Montoya, «Les territoires autochtones face aux industries extractives au Chili : Enjeux environnementaux et modes de résistance communautaire », Éducation relative à l'environnement [En ligne], Volume 12 | 2015, mis en ligne le 20 mai 2015, consulté le 21 février 2020. URL : http://journals.openedition.org/ere/511; DOI : 10.4000/ere.511 


\title{
Les territoires autochtones face aux industries extractives au Chili : Enjeux environnementaux et modes de résistance communautaire
}

\author{
Blaise Pantel et Ximena Cuadra Montoya
}

1 Depuis la fin de la dictature en 1990, le Chili a développé de grands projets industriels extractifs relatifs à l'exploitation minière, forestière, chimique, hydraulique et piscicole. La législation environnementale a largement favorisé ce type de développement économique au détriment d'importants impacts environnementaux et socio-culturels sur les populations locales, notamment sur les communautés et les peuples autochtones. Parallèlement et dans une stratégie de résistance territoriale, se sont déployées des expériences communautaires et citoyennes qui remettent en cause le mode de développement favorisé par les dirigeants du pays.

2 À travers deux exemples, nous souhaitons illustrer comment les actions mises en place par les mouvements autochtones de résistance territoriale constituent un important capital d'expérience. Nous désirons aborder, à travers la théorie des mouvements sociaux, les cycles d'action collective, le développement de nouveaux répertoires d'action ainsi que les dynamiques qui permettent de transformer les structures d'opportunités politiques (Tilly, 1978, 1995 ; Tarrow, 1998 ; Jiménez, 2005). Les liens tissés par ces mouvements de résistance locaux avec d'autres mouvements de résistance à l'échelle nationale et internationale permettent de nouvelles formes d'apprentissage. Nous verrons que les processus de résistance impliquent en effet des espaces plus larges que la seule dimension locale et territorialisée du conflit entre les différents acteurs impliqués.

3 Nous développerons notre propos en trois points : d'abord, quelques données générales sur les peuples autochtones au Chili et le contexte sociopolitique du pays; puis, la présentation de deux expériences communautaires de défense territoriale; enfin, une réflexion sur les nouveaux types et modes d'apprentissage au sein des mouvements de 
résistance. Nous présenterons de pistes de discussion autour de la question autochtone au Chili et de l'approche théorique des mouvements sociaux, dans la perspective de lier ces questions à certains enjeux en matière d'éducation relative à l'environnement.

\section{Les peuples autochtones et le contexte sociopolitique au Chili 1}

4 Aujourd'hui, le Chili reconnaît officiellement neuf peuples autochtones. Selon le recensement de 1992, près d'un million de personnes se sont déclarées autochtones, soit $10,3 \%$ de la population totale. En 2002, ce chiffre a diminué à un peu moins de 700000 personnes, soit $4,6 \%$ de la population totale. Cette baisse significative est principalement due au type de questions qui ont été posées.

5 Selon les données statistiques de l'Enquête de caractérisation socioéconomique nationale (CASEN) qui permet une actualisation plus fréquente que le recensement, la population autochtone du pays est estimée en 2006 à un peu plus d'un million de personnes, soit $6,6 \%$ de la population totale ${ }^{2}$. De cette population autochtone, $87,2 \%$ se déclare mapuche, le peuple autochtone le plus important du pays. Comme il y a vingt ans, on estime aujourd'hui qu'environ $10 \%$ de la population chilienne se définit comme autochtone (Aravena, 2014).

6 En ce qui concerne le contexte socioéconomique du pays, la privatisation des ressources naturelles est le facteur majeur qui soutient le modèle de développement économique chilien. Ce processus de privatisation des ressources, comme l'eau et le sous-sol, se base sur un droit réel d'utilisation que l'État octroie aux particuliers qui le sollicitent. Ce droit d'utilisation des ressources naturelles est équivalant à un droit de propriété à travers un système de concessions. La base juridique du système néolibéral chilien qui assure la privatisation des ressources naturelles repose sur le Code de l'Eau et le Code Minier. Ces lois dites sectorielles bénéficient d'une protection constitutionnelle (Article 19 de la Constitution du Chili).

7 Quant aux peuples autochtones, les accords de Nueva Imperial ont établi en 1989, à la fin de la dictature, trois engagements politiques (Yáñez et Aylwin, 2007) : la création d'une Loi autochtone (Ley Indígena); la ratification de la Convention 169 de l'Organisation Internationale du Travail sur les peuples autochtones et tribaux; une reconnaissance constitutionnelle des peuples autochtones.

8 En 1993 et sous le conglomérat de centre-gauche qui a gouverné le pays jusqu'en 2010, la Ley Indígena est promulguée avec la création de la Corporation Nationale de Développement Indigène (CONADI), un organisme d'État. Sa politique a consisté à promouvoir la culture autochtone et à acheter, à travers un fonds spécial, des terres et des droits de l'eau pour les communautés autochtones. En 1996, une loi environnementale a également été promulguée puisque le Chili n'avait aucun mécanisme régulateur en la matière. En 2010, cette loi a été réformée avec la création d'un ministère. Une des principales critiques de cette réforme environnementale est qu'elle n'a pour seul but de corriger certains problèmes liés au développement de projets industriels. Au-delà d'évaluations techniques, c'est finalement le pouvoir politique (à travers un représentant régional) qui décide de l'adoption de tels projets. La participation citoyenne n'est qu'une formalité administrative sans aucune influence réelle. Quant à la ratification de la Convention 169 de l'Organisation Internationale du 
Travail en 2008, longtemps exigée par les peuples autochtones, elle a requis près de dixhuit années de processus parlementaire. Son application est au cœur des enjeux aujourd'hui, notamment en ce qui concerne la question de la consultation des peuples autochtones. Il n'y a toujours pas de reconnaissance constitutionnelle de cette convention et le Chili reste un des pays d'Amérique du Sud les plus en retard en matière de reconnaissance des droits des peuples autochtones. Si dans la législation chilienne, la Ley Indígena reconnait les autochtones en tant que minorités culturelles, ce statut reste cependant limité en matière d'autonomie et de prise de décision par ces populations (Aylwin, 2002).

\section{Deux expériences de résistance territoriale}

Dans ce contexte politico-économique chilien, les pouvoirs publics ont permis le développement, au cours des deux dernières décennies, d'importants projets industriels à grande échelle dans les domaines de l'exploitation minière, forestière, chimique, hydraulique et piscicole. Le pays se le positionne aujourd'hui comme l'un des moteurs de l'économie latino-américaine. Comme nous l'avons souligné, la législation existante a largement favorisé ce type de développement qui a entraîné de nombreux impacts environnementaux et socioculturels sur les populations locales, notamment sur les communautés et peuples autochtones, et dont a résulté un accroissement des inégalités, l'exclusion sociale, et la paupérisation croissante des populations concernées. Toutefois, peu à peu et dans une stratégie de résistance territoriale, des expériences communautaires et citoyennes se sont développées pour remettre en question le mode de développement du pays. Plusieurs conflits ont alors émergé où la gestion des biens et des ressources naturelles, ainsi que celle des territoires, sont au cœur des débats politiques sur la reconnaissance des peuples et des populations locales, de leurs droits, de leurs savoirs et de leurs cultures.

10 Nous aborderons ici deux cas spécifiques : celui de Mehuin et de Liquiñe/Coñaripe. Il s'agit de deux territoires situés au centre-sud du pays ayant une forte population autochtone, principalement mapuche ${ }^{3}$.

\section{Le projet d'installation d'une canalisation d'évacuation de déchets toxiques vers la localité de Mehuin}

11 Méhuin est une zone côtière habitée de pêcheurs artisanaux et de communautés mapuches. En 1996, la localité prend connaissance de l'existence d'un projet d'évacuation de déchets toxiques par le biais de travailleurs de l'entreprise CELCO chargés des activités d'exploration dans la zone de pêche. Il s'agissait de mettre en place une conduite d'environ 40 kilomètres permettant à l'entreprise de déverser ses déchets toxiques à la mer, avec pour conséquences d'importants impacts environnementaux sur le littoral côtier. Immédiatement, les habitants locaux expulsèrent les travailleurs de l'entreprise de leurs terres et de leurs aires maritimes pour empêcher la réalisation des études techniques et refusèrent de participer aux mécanismes de consultation prévus par la loi environnementale. Comme l'entreprise n'a pas pu faire les études techniques, en 1998 une décision judiciaire empêcha la construction de la canalisation, par manque d'information. Or, en 2006, la proposition initiale de construire cette conduite est de nouveau d'actualité. La communauté locale 
réagit avec une stratégie similaire que celle adoptée précédemment : tout en critiquant les limites du processus de consultation, il s'agissait de rendre impossible la réalisation des études techniques, en raison des impacts irréversibles qu'un tel projet impliquerait sur le littoral, riche en ressources maritimes. Cependant, l'entreprise décide alors d'offrir de l'argent et des compensations à certains syndicats de pêche. Cette stratégie suscita une importante division interne et l'entreprise CELCO put livrer une étude dans le cadre du système d'évaluation environnemental.

12 Face à cette situation de tensions internes, les communautés et syndicats de pêche en résistance décidèrent de former des alliances stratégiques avec le mouvement autochtone à l'échelle nationale. Peu à peu, le conflit est connu à l'échelle internationale comme un conflit de droits autochtones. En 2010, le projet est approuvé par le système d'évaluation environnemental chilien. Les communautés mapuches, avec l'appui de certains syndicats de pêche, décidèrent alors de porter plainte devant la justice réclamant le manque de consultation. Les procès furent perdus, en première instance et face à la Cour Suprême du pays. En 2011, le cas est porté à la Commission interaméricaine des Droits de l'Homme et le processus est actuellement en cours.

\section{Les projets hydroélectriques de Liquiñe/Coñaripe}

En 2006, l'entreprise norvégienne SN Power décida de planifier la réalisation de trois centrales hydroélectriques dans la région de Liquiñe/Coñaripe. Ces projets devaient affecter plusieurs communautés mapuches dans un territoire à fort potentiel écotouristique, en inondant certains territoires et lieux cérémoniels.

En 2006 et 2007, l'entreprise organisa plusieurs rencontres pour expliquer la nature exacte des projets hydroélectriques et surtout pour planifier les études techniques sur le terrain. De manière parallèle, des comités citoyens locaux et les communautés mapuches se sont peu à peu organisés pour empêcher la réalisation de ces études techniques et pour manifester leur refus collectif face à ces projets. Les travailleurs de l'entreprise sont alors expulsés du territoire. En décembre 2007, grâce à l'appui d'une organisation locale de la société civile et d'une association norvégienne qui faisait un suivi du dossier, une petite délégation - avec à sa tête un dirigeant mapuche - effectua un voyage en Norvège. Un fort lobby politique fut alors exercé à travers des rencontres avec des parlementaires norvégiens et les institutions de financement de l'entreprise. Ce voyage à l'international a constitué un précédent puisque l'entreprise s'est engagée à l'époque à cesser ses activités dans le territoire et à consulter les communautés. Par la suite, les responsables de l'entreprise organisèrent des tables rondes pour informer les populations concernées et négocier une entente, sans grand résultat. En 2011, SN Power décida d'abandonner les projets et de céder ses droits de l'eau à un partenaire, l'entreprise australienne Centinela. Cette dernière les céda par la suite à l'entreprise Hidro-Aconcagua qui souhaite aujourd'hui développer de nouveaux projets hydroélectriques dans cette zone.

\section{Nouveaux types et modes d'apprentissage des mouvements de résistance}

Pour examiner les nouveaux types et modes d'action et d'apprentissage au sein des mouvements de résistance en territoires autochtones au Chili, nous utiliserons trois 
concepts clés dérivés de l'approche socio-historique des mouvements sociaux développée initialement par Charles Tilly $(1995 ; 1978)$ et puis entre autres, par Sidney Tarrow (1998): le cycle d'action collective, les répertoires d'action collective et la structure d'opportunités politiques.

En premier lieu, nous considérons que les diverses stratégies de mobilisation font partie d'un cycle d'action collective. Selon Sidney Tarrow (1998), les mouvements sociaux développent des phases d'intensification des conflits face au système social, ce qui provoque une diffusion rapide des demandes, de l'innovation dans les formes de confrontation et une diversification des mécanismes de participation. Nous observons en ce sens que les actions des communautés mapuches, avec des demandes particulières dans des contextes spécifiques, sont des éléments liés au mouvement mapuche en général. Selon nos observations, ce cycle d'action collective se caractérise dans le cas chilien par l'exigence de droits territoriaux et de droits liés aux peuples autochtones, aujourd'hui reconnus à l'échelle internationale et qui s'expriment dans la Convention 169 de l'Organisation Internationale du Travail et la Déclaration des Nations Unies sur les droits des peuples autochtones; ces droits permettent de mobiliser des stratégies autour de revendications liées à l'autodétermination. Les cas que nous avons analysés peuvent certes être étudiés sous l'angle de leurs cycles internes, mais d'une part, ils doivent être compris en relation avec le cycle d'action collective développé par le mouvement mapuche en général et d'autre part, avec le fait que ce dernier est lié à des processus globaux de revendications autochtones. Par ailleurs, ces processus s'inscrivent dans un contexte de développement de projets extractifs et énergétiques à l'échelle mondiale donnant lieu à une pluralité de mouvements de contestation citoyenne et écologiste qui remettent en cause ce modèle, comme c'est le cas au Chili.

17 Pour illustrer cet argument à l'aide des cas présentés, nous soulignons que les communautés de Mehuin dans leurs contestations des années 90 structuraient leur discours politique autour des impacts environnementaux sur la mer et la pêche, alors qu'à partir de 2006, le discours est centré sur la dénonciation des violations d'un territoire autochtone revendiqué. Dans le cas de Liquiñe/Coñaripe, le voyage d'une délégation locale vers le siège social de l'entreprise en Norvège illustre l'emboîtement d'une situation locale dans un questionnement plus global sur les préoccupations relatives à la situation des peuples autochtones et aux projets d'extraction des ressources naturelles. De plus, il a été demandé à l'entreprise de respecter la Convention 169 de l'Organisation Internationale du Travail, alors qu'elle n'était pas encore ratifiée au Chili, mais bien en vigueur en Norvège, lieu d'origine des capitaux.

En second lieu, nous observons un enrichissement du répertoire d'action collective. Les mouvements sociaux ont recours à des formes et des moyens d'action qui ont été utilisés dans des contestations antérieures et qui se sont incorporés à la culture politique, c'est-à-dire qui se sont transmis socialement (Tilly, 1978 ; 1995 ; Traugott, 1995 ; Tarrow, 1998). Mais on observe que si la forme d'action collective peut être héritée, elle peut aussi émerger comme une innovation sociale.

Dans le cas de Mehuin, la stratégie de blocage de l'entreprise pour l'empêcher de réaliser des études techniques a été une innovation qui aujourd'hui s'inscrit dans le répertoire d'actions collectives utilisées par d'autres mouvements de résistance, comme dans le cas de Liquiñe/Coñaripe. D'autre part, l'utilisation d'une stratégie internationale, que ce soit dans le cas de Liquiñe/Coñaripe avec l'exercice d'un lobby 
politique ou bien dans le cas de Mehuin avec une démarche judiciaire, reproduit un mode d'action déjà utilisé par d'autres organisations autochtones du Chili.

Il est important de souligner que pendant ces deux dernières décennies, à travers le travail des organisations autochtones ou bien celui des organisations alliées de la société civile, se sont construits des espaces d'apprentissage collectif et d'échanges d'expériences sur les stratégies locales, nationales et globales. Nous pensons ici à des rencontres, des ateliers, des réunions, des colloques, etc. qui ont permis un véritable partage de connaissances et de savoirs collectifs autour de la question de la résistance territoriale et des modes d'action associés, au-delà de conjonctures spécifiques.

21 En troisième lieu, on observe que les mouvements sociaux mettent à profit certaines opportunités dérivées du contexte politique (Tilly, 1998). Mais également, comme dans les deux cas ici esquissés, les stratégies utilisées peuvent aussi en retour avoir une répercussion sur cette même structure. Dans les cas étudiés, les protagonistes autochtones ont utilisé de manière critique les instruments du droit international, par exemple le droit à la consultation dans le cadre de la Convention 169 de l'Organisation Internationale du Travail, au moment même où ces mouvements de résistance exigeaient sa ratification. Aujourd'hui, au Chili, commence à se constituer une jurisprudence sur la question de la consultation à partir d'autres cas significatifs à ce sujet. En 2012 par exemple, dans le cas de deux projets industriels majeurs, la Cour Suprême du pays a obligé le respect de ce droit à la consultation, paralysant un parc éolien au sud du pays dans une communauté mapuche et l'exploitation d'une mine d'or au nord du pays dans une communauté Diaguita.

\section{Réflexions finales : communautés d'apprentissage au- delà de l'espace local}

Il faut enfin reconnaître que des modes d'apprentissage nouveaux se sont développés au Chili dans le cadre de mouvements de résistance de communautés autochtones face à des projets qui affectent leurs territoires. Des espaces collectifs, autochtones et non autochtones, reliés entre eux, se sont développés, que ce soit à l'échelle locale et interne de ces mouvements, ou à l'échelle nationale et internationale où se constituent des alliances politiques. Peu à peu commence à se construire un réseau de relations qui pourrait être considéré comme une communauté d'apprentissage transnationale où se partagent des stratégies et se développent des discours politiques. Nous adoptons ici le concept de communauté d'apprentissage pour caractériser un processus de construction collective de savoirs et de connaissances qui permet aux membres d'un groupe de s'approprier leur réalité, de créer des outils pour transformer les situations qui sont à la base d'injustices ou les contextes défavorables face auxquels ils se mobilisent et qui impliquent des enjeux socio-environnementaux et culturels (Orellana, $2002 ; 2005)$.

Les mouvements locaux de résistance au Chili peuvent être ainsi considérés comme des communautés d'apprentissage qui interagissent avec d'autres regroupements ou associations, où les participants partagent des savoirs expérientiels et développent des compétences nouvelles. De tels mouvements créent ou renforcent un sentiment d'appartenance communautaire et entraînent une réappropriation du territoire 
partagé. Ainsi, après de nombreuses rencontres d'échange et de réflexion, la communauté de Liquiñe adopte une stratégie utilisée à Mehuin.

À l'échelle internationale, nous observons également l'adoption de stratégies qui prennent en compte une dimension plus vaste de la communauté d'apprentissage et où se développe un militantisme ou bien un activisme à caractère transnational. Dans les deux cas présentés, il y a des militants en dehors du Chili qui ont appuyé les stratégies locales, qui ont mobilisé des ressources stratégiques pour le développement de la mobilisation locale et nationale, et qui ont fait partie d'un processus collectif de définition d'actions à entreprendre. En ce sens, il y aurait intérêt à explorer cette nouvelle dimension des mouvements sociaux, celle de leur étendue géo-politique favorisée aujourd'hui par les nouveaux modes de communication - qui permet entre autres d'enrichir les dynamiques d'apprentissage social.

Le concept de cycle d'action collective ouvre ainsi d'importantes perspectives pour examiner les mouvements sociaux et les processus d'apprentissage qui y sont associés, en les inscrivant dans un espace-temps plus vaste, ce qui permet de ne pas les limiter à des réalités statiques et figées. De plus, ce cadre de référence nous aide à identifier des critères importants pour la sélection, l'usage, la consolidation ou l'abandon de certains modes d'action qui peuvent influencer la structure d'opportunités politiques.

Par ailleurs, il nous importe de mettre en évidence qu'à la base des mouvements sociaux, se développement des processus d'apprentissage d'ordre collectif. Et plus spécifiquement, à partir des deux cas analysés, nous souhaitons ajouter à la réflexion sur le déploiement possible et souhaité d'une communauté d'apprentissage au niveau transnational autour d'enjeux liés à l'environnement et l'aménagement du territoire.

\section{BIBLIOGRAPHIE}

Aravena Reyes, A. (2014). Identidad indígena en Chile en contexto de migración, urbanización y globalización. Amérique Latine Histoire et Mémoire. Les Cahiers ALHIM, 27. Récupéré du site de la revue http://alhim.revues.org/4942

Aylwin, J. (2002). Políticas públicas y pueblos indígenas : el caso de la política de tierras del Estado chileno y el pueblo mapuche. Dans Communication présentée à l'University of Texas, Center for Latin American Social Policy (CLASPO), États-Unis. Récupéré de http://lanic.utexas.edu/project/ laoap/claspo/dt/0006.pdf

Jiménez Sánchez, M. (2005). El impacto político de los movimientos sociales. Un estudio de la protesta ambiental en España. Centro de Investigaciones Sociológicas (CIS). Madrid : Siglo XXI.

Observatorio Ciudadano (Comp.). (2009). Los derechos humanos en Chile : la evaluación de la sociedad civil, los pueblos indígenas y las Naciones Unidas. Temuco : Observatorio de derechos de los pueblos indígenas.

Orellana, I. (2002). La communauté d'apprentissage en éducation relative à l'environnement : signification, dynamique, enjeux (thèse de doctorat non publiée). Université du Québec à Montréal, Montréal, Canada. 
Orellana, I. (2005). L'émergence de la communauté d'apprentissage ou l'acte de recréer des relations dialogiques et dialectiques de transformation du rapport au milieu de vie. Dans Lucie Sauvé, Isabel Orellana et Étienne van Steenberghe (dir.), Éducation et environnement. Un croisement de savoirs (p. 67-84). Québec : Cahiers scientifiques de l'Acfas, 104

Tarrow, S., (1998). Power in movement. Cambridge : Cambridge University Press.

Tarrows, S. (2005). The new transnational activism. New York : Cambridge University Press.

Tilly, C. (1978). From mobilization to revolution. Reading, Massachusetts : Addison-Wesley. Tilly, C. (1995). Popular Contention in Great Britain. Cambridge, Mass. : Harvard University Press.

Traugott, M. (1995). Barricades as repertoires : Continuities and discontinuities in the history of French contention. Dans Traugott, M. (dir.), Repertoires and cycles of collective action (p. 43-56). Durham-Londres : Duke University Press.

Yáñez, N. et Aylwin, J. (Ed.) (2007). El gobierno de Lagos, los pueblos indígenas y el « nuevo trato».

Las paradojas de la democracia chilena. Santiago de Chile : Lom.

\section{NOTES}

1. Pour plus d'information sur les statistiques du pays : www.ine.cl/canales/chile_estadistico/ estadisticas_sociales_culturales/etnias/etnias.php

2. Données statistiques de l'Enquête de Caractérisation Socioéconomique Nationale (CASEN) : www.ministeriodesarrollosocial.gob.cl/casen/publicaciones.html

3. Nous utiliserons la référence suivante pour les deux cas : Observatorio Ciudadano (Comp.) (2009). Los derechos humanos en Chile : la evaluación de la sociedad civil, los pueblos indígenas y las Naciones Unidas. Temuco : Observatorio de derechos de los pueblos indígenas.

\section{INDEX}

Mots-clés : territoire, territoire autochtone, projet énergétique, extractivisme, déchets, résistance, résistance communautaire, peuple autochtone

Keywords : territory, indigenous territory, energy project, extractivism, waste, resistance, community resistance, indigenous people

Index géographique : Chili, Amérique du Sud

\section{AUTEURS}

\section{BLAISE PANTEL}

Titulaire d'un DEA en Études sur l'Amérique latine à l'IPEALT de l'Université de Toulouse-leMirail (France) et d'une Maîtrise en Sociologie complétée à la même université. Membre de l'Observatoire Citoyen au Chili, il a été coordonnateur du Programme de Droits Autochtones 
(2008-2010). Il est actuellement professeur au Département de sociologie et science politique et directeur du programme de sociologie à l'Université Catholique de Temuco, au Chili.

\section{XIMENA CUADRA MONTOYA}

Titulaire d'une Maîtrise en Sociologie de l'Université de Barcelone (Espagne) et d'une Maîtrise en science politique de l'Université du Québec à Montréal - UQAM. Elle poursuit actuellement un doctorat en science politique à l'UQAM. Elle est membre fondatrice de l'Observatoire Citoyen et a dirigé plusieurs projets de coopération en matière de droits humains et autochtones au Chili. 